\title{
Least Reflexive Points of Relations
}

\author{
Jules Desharnais $^{1 *}$ and Bernhard Möller ${ }^{2}$ \\ ${ }^{1}$ Département d'Informatique, Université Laval, Québec, QC, G1K 7P4 Canada \\ Jules. Desharnais@ift.ulaval .ca \\ ${ }^{2}$ Institut für Informatik, Universität Augsburg, D-86135 Augsburg, Germany \\ Bernhard.Moeller@informatik.uni-augsburg.de
}

Summary. Assume a partially ordered set $(S, \leq)$ and a relation $R$ on $S$. We consider various sets of conditions in order to determine whether they ensure the existence of a least reflexive point, that is, a least $x$ such that $x R x$. This is a generalization of the problem of determining the least fixed point of a function and the conditions under which it exists. To motivate the investigation we first present a theorem by Cai and Paige giving conditions under which iterating $R$ from the bottom element necessarily leads to a minimal reflexive point; the proof is by a concise relation-algebraic calculation. Then, we assume a complete lattice and exhibit sufficient conditions, depending on whether $R$ is partial or not, for the existence of a least reflexive point. Further results concern the structure of the set of all reflexive points; among other results we give a sufficient condition for these to form a complete lattice, thus generalizing Tarski's classical result to the nondeterministic case.

Key words:partial order, fixed point, least reflexive point, greatest reflexive point, lattice, relation, inflationary relation.

\section{Introduction}

Iterative and recursive processes are at the center of computer science. The mathematical background is the theory of (least) fixed points and is well understood in the case where the iteration can be described by a (total) function $[4,10]$.

Much less is known about fixed points of relations. The problem Find the least $x$ related to itself was stated to the first author in these terms by Robert Paige in 1992, at the 44th meeting of the IFIP Working Group 2.1 (Algorithmic Languages and Calculi), which was held in Augsburg, Germany, and was organized by the second author. This problem has its origin in the work presented in [3]. There, the authors are concerned with the construction of efficient algorithms expressed in a language using set-theoretic queries augmented with nondeterministic minimal and maximal fixed point queries (the deterministic case is treated in an earlier paper [2]).

Let us state the problem more precisely. Consider a partially ordered set $(S, \leq)$ and a binary relation $R$ on $S$. A reflexive point of $R$ is an element $x \in S$ such that $x R x$. In the sequel we give conditions under which $R$ has a least reflexive point and investigate the structure of the set of all reflexive points of $R$. It turns out that under a suitable relational generalization of the property of monotonicity, the set of reflexive points even forms a complete lattice, so that Tarski's classical results generalize nicely to the nondeterministic case.

As a starting point, we present in Section 2 a theorem by Cai and Paige [3] giving conditions under which iterating $R$ from the bottom element of the partial order necessarily leads to a minimal reflexive point; this theorem is based on the notion of an inflationary relation.

In Section 3, we first define four conditions generalizing monotonicity. Then, we examine which combinations of these, if any, are sufficient to ensure the existence of a least reflexive point; total and partial relations are tackled separately. In Section 4, we uncover some

\footnotetext{
* This research was supported by a grant from NSERC (Natural Sciences and Engineering Research Council of Canada).
} 
additional structure on the set of reflexive points. In Section 5, we use duality principles to present analogous results about greatest reflexive points. In Section 6, we study another set of four possible generalizations of monotonicity and we explain their relationship with the four conditions of Section 3.

We conclude with an evaluation of the results achieved and directions for future research. There are also two appendices. Appendix A gives graphical representations of the lattices and relations used as examples in the paper. They are grouped together to facilitate comparisons. The diagrams are labelled alphabetically. In the text, we refer to these diagrams by "Appendix A(a)", "Appendix A(b)", etc. Appendix B contains examples for all possible combinations of the conditions from Section 3 and all possible combinations of those of Section 6. Most proofs are omitted. They can be found in $[5,6]$.

\section{Reflexive Points of Inflationary Relations}

As stated in the introduction, Cai and Paige [3] are concerned with the construction of efficient algorithms expressed in a language using set-theoretic queries augmented with nondeterministic minimal and maximal fixed point queries. A typical algorithm is one that finds a maximal independent set of vertices of an undirected graph. A maximal independent set of an undirected graph $(V, E)$ is a subset $U \subseteq V$ such that for any $(u, v) \in E$, at most one of $u$ and $v$ is in $U$ and, for any $u \in V-U$, there is a vertex $v \in U$ such that $(u, v) \in E$.

Consider the following graph. It has two maximal independent sets of vertices, namely $\{1,3\}$ and $\{2,4\}$.

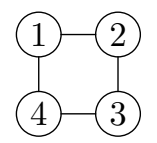

An algorithm incrementally building a maximal independent set would initially choose any vertex and add new vertices, provided that this preserves independence, until a fixed point is reached where no more vertices can be added. Here is such an algorithm: ${ }^{3}$

$$
\begin{aligned}
& U:=\emptyset \text {; } \\
& \text { while } \exists(v: v \in V-U: U \cup\{v\} \text { is independent) do } \\
& \qquad U:=U \cup \ni\{v \mid U \cup\{v\} \text { is independent }\}
\end{aligned}
$$

The expression $\ni S$ denotes an arbitrary element from the nonempty set $S$. The following relation $R$ on the powerset $\mathcal{P}\{1,2,3,4\}$ is the relation computed by the body of the nondeterministic loop, i.e., the set of pairs $\left(U_{1}, U_{2}\right)$ such that $U_{2}$ is a possible value of variable $U$ after one execution of the body of the loop if $U_{1}$ is the value of $U$ before the execution.

$$
R:=\left\{\begin{array}{lll}
(\emptyset,\{1\}) & (\{1,2\},\{1,2\}) & (\{1,2,3\},\{1,2,3\}) \\
(\emptyset,\{2\}) & (\{1,3\},\{1,3\}) & (\{1,2,4\},\{1,2,4\}) \\
(\emptyset,\{3\}) & (\{1,4\},\{1,4\}) & (\{1,3,4\},\{1,3,4\}) \\
(\emptyset,\{4\}) & (\{2,3\},\{2,3\}) & (\{2,3,4\},\{2,3,4\}) \\
(\{1\},\{1,3\}) & (\{2,4\},\{2,4\}) & (\{1,2,3,4\},\{1,2,3,4\}) \\
(\{2\},\{2,4\}) & (\{3,4\},\{3,4\}) & \\
(\{3\},\{1,3\}) & & \\
(\{4\},\{2,4\}) &
\end{array}\right\}
$$

This relation has no least reflexive point, but it has many minimal ones, namely all the subsets of $\{1,2,3,4\}$ with exactly two elements.

One interesting property of relation $R$ in (1) is that any path starting at $\emptyset$ necessarily leads to a minimal reflexive point. Hence, one can build a minimal reflexive point iteratively starting from $\emptyset$ - this is what the above algorithm does.

We now give sufficient conditions that ensure this property.

\footnotetext{
${ }^{3}$ Quantifiers have three arguments: a list of variables, the domain over which the quantification applies, and the quantified expression; for instance, $\forall(x: P: Q)$ is read "for all $x$ satisfying $P$, $Q$ holds", or "for all $x, P \Rightarrow Q$ ", while $\exists(x: P: Q)$ is read "there exists an $x$ satisfying $P$ and $Q$ ". When the second argument is true, it is omitted.
} 
By $V$ and $I$ we denote the universal and identity relations, respectively. The complement of a set or relation $R$ is denoted by $\bar{R}$. The composition (or relative product) of two relations $Q$ and $R$ is defined by $Q ; R:=\{(s, u) \mid \exists(t:: s Q t$ and $t R u)\}$. As usual, $R^{*}$ denotes the reflexive and transitive closure of $R$. The converse of a relation $R$ is defined by $R^{\longleftarrow}:=\{(s, t) \mid t R s\}$.

Definition 1 Let $(S, \leq, \perp)$ be a partial order with least element $\perp$. We say that a relation $R$ on $S$ is inflationary [3] iff $R$ is total and included in $\leq$, i.e., $R ; V=V$ and $R \subseteq \leq$. In elementwise terms this means $\forall(x:: \exists(y:: x R y))$ and $\forall(x:: \forall(y: x R y: x \leq y))$.

A relation $R$ is progressively finite iff there is no infinite chain $s_{0}, s_{1}, s_{2}, \ldots$, such that $\left(s_{i}, s_{i+1}\right) \in R$, for all $i \geq 0[9]$.

Because the notion of well-foundedness is often used to characterize relations that do not have infinite chains, we remark that $R$ is progressively finite iff its converse is well-founded.

Proposition 1 Let $Q$ and $R$ be relations and $f(X):=R ; X \cup Q$.

1. If $R$ is progressively finite, then $f$ has a unique fixed point, viz. $R^{*} ; Q$ [1].

2. If $Q \subseteq R$ and $R$ is progressively finite, then $Q$ is progressively finite.

Is is shown in [3] that, for an inflationary relation $R$ on a progressively finite order, iteration from an arbitrary element necessarily leads to a reflexive point. To state this in relationalgebraic terms we first observe that $R \cap I$ is a partial identity relation characterizing the set of reflexive points of $R$. Hence we have $x R^{*} ;(R \cap I) y$ iff from $x$ we can reach a reflexive point $y$ by iterating $R$. The claim follows if we can show that this relation is total, which is expressed by $R^{*} ;(R \cap I) ; V=V$.

Theorem 1 Let $(S, \leq)$ be a partial order such that $<$ is progressively finite. Let $R$ be a relation on $S$. If $R$ is inflationary, then $R^{*} ;(R \cap I) ; V=V$.

If $(S, \leq)$ has a least element $\perp$ then $\perp$ is a natural starting point for the iteration of $R$.

The relation $R$ in (1) is inflationary, using the ordering $\subseteq$ on $\mathcal{P}\{1,2,3,4\}$, and $\subset$ is progressively finite. This is why from any subset of $\{1,2,3,4\}$ there is a path by $R$ to a reflexive subset of $\{1,2,3,4\}$.

We now present a different example, where relation $R$ still satisfies the preconditions of Theorem 1 , and also has a least reflexive point. The lattice is $\mathcal{P}\{1,2,3\}$ with the inclusion ordering. The relation $R$ is the Hasse diagram of $\subset$ plus the pair $(\{1,2,3\},\{1,2,3\})$, that is,

$$
R:=\left\{\begin{array}{lll}
(\emptyset,\{1\}) & (\{1\},\{1,2\}) & (\{1,2\},\{1,2,3\}) \\
(\emptyset,\{2\}) & (\{1\},\{1,3\}) & (\{1,3\},\{1,2,3\}) \\
(\emptyset,\{3\}) & (\{2\},\{1,2\}) & (\{2,3\},\{1,2,3\}) \\
& (\{2\},\{2,3\}) & (\{1,2,3\},\{1,2,3\}) \\
& (\{3\},\{1,3\}) & \\
& (\{3\},\{2,3\}) &
\end{array}\right\}
$$

This relation could correspond to an (extremely simple) algorithm that, given a set $T$, adds to $T$ an element not in $T$, if there is any. Note that $R$ is inflationary and that $\subset$ is progressively finite. Thus Theorem 1 applies and explains why from any subset of $\{1,2,3\}$ there is a path to the unique reflexive point $\{1,2,3\}$.

\section{Four Conditions Generalizing Monotonicity}

In this section, we generalize the classical fixed point theory of monotonic functions on complete lattices to the relational case. Therefore, we assume the partial order to be a complete lattice $(S, \sqcap, \sqcup, \perp, \top, \leq)$. Letting $R$ be a binary relation on $S$, we give in Theorem 2 a sufficient condition implying the existence of a least reflexive point for $R$ when $R$ is a total relation. A consequence of this lemma is Theorem 3 in Section 4, which shows that under the same condition the set of reflexive points of total relations is a complete lattice. We deal with partial relations in Section 3.4.

We denote, for relation $R$ and element $x$, by $x R:=\{y \mid x R y\}$ the set of images of $x$, and, for any $T \subseteq S$, by $\sqcap T$ and $\sqcup T$ the meet and join, respectively, of the elements in $T$. With these conventions we define the following notations: 


$$
\begin{aligned}
& \text { (a) } \quad m_{\sqcap}:=\sqcap\{x \mid \sqcap x R \leq x\}, \\
& \text { (b) } m_{\sqcup}:=\sqcap\{x \mid \sqcup x R \leq x\} .
\end{aligned}
$$

The elements $m_{\sqcap}$ and $m_{\sqcup}$ generalize the notion of least prefixed point of a function; indeed, for a total function $R$, we have $\sqcap x R=\sqcup x R=$ the unique image of $x$, so that $m_{\sqcap}=m_{\sqcup}=$ the least prefixed point of $R$.

\subsection{Monotonicity of Relations}

In the theory of fixed points of functions, monotonic functions play a major role and we seek generalizations of this notion to the case of relations. The following are four natural conditions that can be imposed on $R$ :

$$
\begin{aligned}
& \text { (a) } \forall(x, y: x<y: \sqcap x R \leq \sqcap y R), \\
& \text { (b) } \forall(x, y: x<y: \sqcap x R \leq \sqcup y R), \\
& \text { (c) } \forall(x, y: x<y: \sqcup x R \leq \sqcap y R), \\
& \text { (d) } \forall(x, y: x<y: \sqcup x R \leq \sqcup y R) .
\end{aligned}
$$

These are natural conditions because they all constrain in some way how the "packet" of images of $x$ increases with increasing $x$, by saying how the lower and upper bounds of these images increase. When $R$ is a total function, all are equivalent and they all state that $R$ is monotonic (due to $\sqcap x R=\sqcup x R=$ the unique image of $x$ ).

A total function is an extreme case of a relation. There are two more relaxed cases: that of total relations and that of partial functions (functions for short, in the sequel). If relation $R$ is total, then $\sqcap x R \leq \sqcup x R$, for all $x$. If $R$ is functional (i.e., is a partial function), then $\sqcup x R \leq \sqcap x R$. This is why we obtain the following implications between Conditions 4(a,b,c,d).

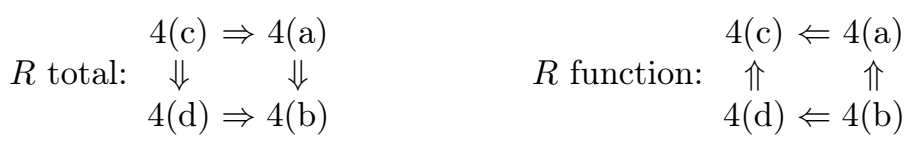

From these diagrams, one can deduce that if $R$ is a total function, all four Conditions $4(\mathrm{a}, \mathrm{b}, \mathrm{c}, \mathrm{d})$ are equivalent, as mentioned above.

Although Conditions 4(a,b,c,d) are not independent for the special kinds of relations mentioned above, in the general case they are, as the following examples show.

1. Condition 4(a) does not follow from (the conjunction of) 4(b,c,d). Take $S$ to be the lattice $\{\perp, \mathrm{a}, \mathrm{b}, \top\}$ with ordering $\perp<\mathrm{a}<\mathrm{T}$ and $\perp<\mathrm{b}<\mathrm{T}$ (see Appendix $\mathrm{A}(\mathrm{a})$ ), and

$$
R:=\{(\mathrm{a}, \mathrm{a}),(\mathrm{a}, \mathrm{b}),(\mathrm{b}, \mathrm{a}),(\mathrm{b}, \mathrm{b}),(\mathrm{T}, \mathrm{\top})\} .
$$

2. Condition $4(\mathrm{~b})$ does not follow from $4(\mathrm{a}, \mathrm{c}, \mathrm{d})$. This can be seen by taking the lattice $\{\perp, \top\}$ with ordering $\perp<\top$ (see Appendix A(b)), and the empty relation

$$
R:=\emptyset \text {. }
$$

3. Condition 4 (c) does not follow from $4(\mathrm{a}, \mathrm{b}, \mathrm{d})$. Take $S$ to be the lattice $\{\perp, \mathrm{a}, \mathrm{b}, \top\}$ with ordering $\perp<\mathrm{a}<\mathrm{T}$ and $\perp<\mathrm{b}<\mathrm{T}$ (see Appendix $\mathrm{A}(\mathrm{c})$ ), and

$$
R:=\{(\perp, \mathrm{a}),(\perp, \mathrm{b}),(\mathrm{a}, \perp),(\mathrm{a}, \top),(\mathrm{b}, \perp),(\mathrm{b}, \top),(\top, \mathrm{a}),(\mathrm{T}, \mathrm{b})\} .
$$

4. Condition 4(d) does not follow from 4(a,b,c). Take $S$ to be the lattice $\{\perp, \top\}$ with ordering $\perp<T$ (see Appendix $\mathrm{A}(\mathrm{d})$ ), and

$$
R:=\{(\perp, \perp),(\perp, \top)\}
$$

In fact, the independence is even more "complete": there are examples for all 16 possible combinations of $4(\mathrm{a}, \mathrm{b}, \mathrm{c}, \mathrm{d})$ (see Appendix B).

As is easily seen, $4(\mathrm{a})$ is equivalent to $x \leq y \Rightarrow \sqcap x R \leq \sqcap y R$, for all $x$ and $y$; this means that the function $(x:: \sqcap x R)$ is monotonic. Similarly, from 4(d), we get that $(x:: \sqcup x R)$ is monotonic. Because we assume a complete lattice, $m_{\sqcap}$ and $m_{\sqcup}$ are the least fixed points of $(x:: \sqcap x R)$ and $(x:: \sqcup x R)$, respectively:
(a) Condition 4(a) implies $\sqcap m_{\sqcap} R=m_{\sqcap}$,
(b) Condition 4(d) implies $\sqcup m_{\sqcup} R=m_{\sqcup}$. 


\subsection{A Stronger Set of Conditions}

One may wonder why in $4(\mathrm{a}, \mathrm{b}, \mathrm{c}, \mathrm{d})$ we did not use $x \leq y$ instead of $x<y$, which would give
(a) $\forall(x, y: x \leq y: \sqcap x R \leq \sqcap y R)$
(b) $\forall(x, y: x \leq y: \sqcap x R \leq \sqcup y R)$,
(c) $\forall(x, y: x \leq y: \sqcup x R \leq \sqcap y R)$,
(d) $\forall(x, y: x \leq y: \sqcup x R \leq \sqcup y R)$.

This is because of the following relationship between these properties:

$$
\begin{array}{ll}
11(\mathrm{a}) \Leftrightarrow 4(\mathrm{a}), & 11(\mathrm{c}) \Leftrightarrow 4(\mathrm{c}) \text { and } R \text { functional, } \\
11(\mathrm{~b}) \Leftrightarrow 4(\mathrm{~b}) \text { and } R \text { total }(\text { if } \top \neq \perp), & 11(\mathrm{~d}) \Leftrightarrow 4(\mathrm{~d}) .
\end{array}
$$

The first and last equivalences are easy to see. The proof of the second one is based on the observation that if the set of images of $x$ is empty, then $\Pi x R \leq \sqcup x R \Leftrightarrow \top \leq \perp$, and the third equivalence is due to the fact that $\sqcup x R \leq \sqcap x R$ holds only if the set of images of $x$ contains at most one element.

Since Conditions $11(\mathrm{~b}, \mathrm{c})$ are too strong, it is better to use $4(\mathrm{a}, \mathrm{b}, \mathrm{c}, \mathrm{d})$ and add totality or functionality only as needed.

\subsection{Reflexive Points of Total Relations}

In this and the following subsection, we deal separately with total relations and partial relations, because this gives a clearer picture of the problem. We begin with the simpler case and assume in this section that relation $R$ is total. Before showing the main result (Theorem 2), we need a lemma.

Lemma 1 Let $R$ be a total relation satisfying Condition $4(\mathrm{c})$. Then $m_{\sqcup} R=\left\{m_{\sqcup}\right\}$. In particular, $m_{\sqcup}$ is a reflexive point of $R$.

One may wonder whether $m_{\sqcap}$ is also a reflexive point under the same conditions (totality $+4(\mathrm{c}))$. The following relation, on the lattice $\{\perp, \mathrm{a}, \mathrm{b}, \mathrm{c}, \top\}$ with ordering $\perp<\mathrm{a}<\mathrm{c}<\mathrm{T}$ and $\perp<\mathrm{b}<\mathrm{c}<\mathrm{T}$, shows that this is not the case (Appendix A(e)). For this relation, $m_{\sqcap}=\perp$.

And here is the main result of this section.

$$
R:=\{(\perp, \mathrm{a}),(\perp, \mathrm{b}),(\mathrm{a}, \mathrm{c}),(\mathrm{b}, \mathrm{c}),(\mathrm{c}, \mathrm{c}),(\top, \top)\}
$$

Theorem 2 Let $R$ be a total relation and assume Condition 4(c). Then $R$ has a least reflexive point, viz., $l:=\sqcap C$, where $C:=\{x \mid x R x\}$.

The relation given in (13) is an example of a total relation that satisfies the precondition of Theorem 2 (i.e., Condition 4(c)). For another, less trivial, example of a relation satisfying 4(c), see (18). Condition 4(c) is very strong, since it implies 4(a,b,c,d) (see (5)).

One can check that the relation (1) of Section 2 satisfies none of the Conditions 4 (a,b,c,d) (for instance, note that $\{1\} \subseteq\{1,2\}$ while $\bigcap\{1\} R=\bigcup\{1\} R=\{1,3\}$ and $\bigcap\{1,2\} R=$ $\bigcup\{1,2\} R=\{1,2\})$. Hence, it is not too surprising that there is no least reflexive point.

We conclude this subsection on the case of total relations with the remark that Condition 4(c) in Theorem 2 cannot be relaxed to a weaker combination of $4(\mathrm{a}, \mathrm{b}, \mathrm{d})$. In (8) we see an example of a total relation that satisfies all of $4(\mathrm{a}, \mathrm{b}, \mathrm{d})$ and that has no reflexive point. On the other hand, relation $R$ in (2) satisfies Conditions 4(a,b,d) but does not satisfy 4(c), while it has a unique reflexive point and thus a least one. So Conditions $4(\mathrm{a}, \mathrm{b}, \mathrm{c}, \mathrm{d})$ do not cover all possible situations. This illustrates the need for other conditions, such as those presented in Section 2.

\subsection{Reflexive Points of Partial Relations}

We suppose here that $R$ is not total, i.e., there is an $s \in S$ such that $s R=\emptyset$. As we will see, this introduces a strong constraint, because $\sqcap s R=\prod \emptyset=\top$ and $\sqcup s R=\sqcup \emptyset=\perp$. We exclude the trivial case where the lattice $S$ contains only one element, since in this case the only partial relation is $\emptyset$, and it has no reflexive point. So, assume $\perp \neq T$.

We could show that the conjunction of Conditions $4(\mathrm{a}, \mathrm{b}, \mathrm{c}, \mathrm{d})$ is sufficient to ensure the existence of a least reflexive point. However, this is a bit too strong. We start by exhibiting combinations of Conditions $4(\mathrm{a}, \mathrm{b}, \mathrm{c}, \mathrm{d})$ that do not ensure the existence of a least reflexive point. This will help pinpointing the essential conditions. 
- The relation $R$ in (6) has three reflexive points, but no least one. This relation satisfies $4(\mathrm{~b}, \mathrm{c}, \mathrm{d})$, but not $4(\mathrm{a})$. This shows that $4(\mathrm{a})$ is essential.

- The relation $R$ in (7) has no reflexive point, hence no least one. This relation satisfies $4(\mathrm{a}, \mathrm{c}, \mathrm{d})$, but not $4(\mathrm{~b})$. This shows that $4(\mathrm{~b})$ is essential.

Thus, 4(a,b) are essential. However, they are not sufficient. The relation

$$
R:=\{(\perp, \mathrm{a}),(\perp, \mathrm{b}),(\mathrm{a}, \mathrm{a}),(\mathrm{a}, \mathrm{b}),(\mathrm{b}, \mathrm{a}),(\mathrm{b}, \mathrm{b})\}
$$

on the lattice $\{\perp, \mathrm{a}, \mathrm{b}, \top\}$, with ordering $\perp<\mathrm{a}<\top$ and $\perp<\mathrm{b}<\top$, satisfies 4 (a,b) (it satisfies none of $4(\mathrm{c}, \mathrm{d}))$ and has no least reflexive point. See Appendix A(f).

We will show that each of the combinations $4(\mathrm{a}, \mathrm{b}, \mathrm{c})$ and $4(\mathrm{a}, \mathrm{b}, \mathrm{d})$ is sufficient. Before dealing with the first one, we derive a consequence of Conditions $4(\mathrm{a}, \mathrm{b})$.

Lemma 2 Let $R$ be a relation satisfying $4(\mathrm{a}, \mathrm{b})$ and let $s$ be such that $s R=\emptyset$. Then

$$
\forall(y: s<y: y R=\{\top\}) \text {. }
$$

Note that Condition 4(b) implies that any two elements that are not in the domain of $R$ are not related by $\leq$.

Proposition 2 Let $R$ be a partial relation satisfying $4(\mathrm{a}, \mathrm{b}, \mathrm{c})$. Then $R$ has a least reflexive point.

In (9), we have already given an example of a partial relation that satisfies $4(\mathrm{a}, \mathrm{b}, \mathrm{c})$ but not 4(d). Another example is the lattice $\{\perp, \mathrm{a}, \mathrm{b}, \mathrm{c}, \mathrm{d}, \mathrm{e}, \mathrm{f}, \mathrm{g}, \top\}$, with ordering

and relation

$$
\perp<\mathrm{a}<\mathrm{T}, \perp<\mathrm{b}<\mathrm{d}<\mathrm{e}<\mathrm{g}<\mathrm{T} \text { and } \perp<\mathrm{c}<\mathrm{d}<\mathrm{f}<\mathrm{g}
$$

$$
\{(\perp, b),(\perp, c),(b, d),(c, d),(d, d),(e, e),(f, f),(g, g),(\top, \top)\} .
$$

See Appendix A(g). Note that the least reflexive point is $d$, which means that the constraints $4(a, b, c)$ may lead to a somewhat more interesting situation than the next case that we analyze, where the least reflexive point is always one of $\perp$ or $T$.

Now we tackle combination $4(\mathrm{a}, \mathrm{b}, \mathrm{d})$ and state a lemma similar to Lemma 2 :

Lemma 3 Let $R$ be a relation satisfying $4(\mathrm{~b}, \mathrm{~d})$ and let $s$ be such that $s R=\emptyset$. Then

$$
\forall(x: x<s: x R=\{\perp\}) \text {. }
$$

Now we obtain

Proposition 3 Let $R$ be a partial relation satisfying $4(\mathrm{a}, \mathrm{b}, \mathrm{d})$. Then $R$ has a least reflexive point, which is either $\perp$ or $T$.

Proof: Let $s$ be such that $s R=\emptyset$. On the basis of Lemma 2 and Lemma 3, we distinguish three cases according to the value of $s$.

1. $s=\perp$ : then $\perp R=\emptyset$ and $t R=\{\top\}$ for every $t \neq \perp$. There is a unique reflexive point, namely $T$, which is thus the least and greatest reflexive point.

2. $s=\top$ : then $\top R=\emptyset$ and $t R=\{\perp\}$ for every $t \neq \top$. There is a unique reflexive point, namely $\perp$, which is thus the least and greatest reflexive point.

3. $s \neq \perp$ and $s \neq \top$ : then $\perp R=\{\perp\}$ and $\top R=\{\top\}$. There is thus a least reflexive point, $\perp$, and a greatest reflexive one, $T$.

In case 3 of the proof of the previous proposition, there might be other reflexive points. As an example, take $S$ to be the lattice $\{\perp, \mathrm{a}, \mathrm{b}, \top\}$ with ordering $\perp<\mathrm{a}<\top$ and $\perp<\mathrm{b}<\top$, and the partial function

Here, $s=$ a. See Appendix A(h).

$$
R:=\{(\perp, \perp),(\mathrm{b}, \mathrm{b}),(\top, \top)\} .
$$

And now an example of a partial relation that satisfies $4(\mathrm{a}, \mathrm{b}, \mathrm{d})$ but does not satisfy $4(\mathrm{c})$ : the lattice is $\{\perp, \mathrm{a}, \mathrm{b}, \mathrm{c}, \mathrm{d}, \mathrm{e}, \top\}$, with ordering $\perp<\mathrm{a}<\mathrm{T}, \perp<\mathrm{b}<\mathrm{c}<\mathrm{e}<\mathrm{T}$ and $\mathrm{b}<\mathrm{d}<\mathrm{e}$, and the relation is

$$
\{(\perp, \perp),(b, c),(b, d),(c, b),(c, e),(d, b),(d, e),(e, c),(e, d),(T, T)\} .
$$

See Appendix A(i). Note that the subrelation on elements $\{b, c, d, e\}$ is the same (modulo renaming) as the one in (8), which was used as a total relation illustrating that $4(\mathrm{a}, \mathrm{b}, \mathrm{d})$ does not imply $4(\mathrm{c})$. 


\section{Insights on the Structure of the Set of Reflexive Points}

\subsection{The Roles of $m_{\sqcap}$ and $m_{\sqcup}$}

The next proposition shows that every reflexive point is above $m_{\sqcap}$.

Proposition $4 \forall\left(x: x R x: m_{\sqcap} \leq x\right)$.

Based on the previous proposition, the next proposition shows that the reflexive points between $m_{\sqcap}$ and $m_{\sqcup}$ are linearly ordered; this only requires Condition $4(\mathrm{c})$. The relation $R$ need not be total.

Proposition 5 Assume Condition 4(c). Then

$$
\forall\left(x: x R x \text { and } y R y: x \leq y \text { or } y \leq x \text { or }\left(m_{\sqcup} \leq x \text { and } m_{\sqcup} \leq y\right)\right) \text {. }
$$

There can be an infinite number of reflexive points between $m_{\sqcap}$ and $m_{\sqcup}$. Let $S$ be the lattice $\mathbf{N} \cup\{\infty\}$, with the usual ordering, where $\mathbf{N}$ is the set of natural numbers. Take

$$
R:=\{(m, n) \mid m=n \text { or }(m \in \mathbf{N} \text { and } n=m+1)\} .
$$

One can check that $R$ satisfies $4(\mathrm{c}), m_{\sqcap}=0, m_{\sqcup}=\infty$, and that every element of $S$ is related to itself, and thus 0 is the least reflexive point. See Appendix $A(j)$.

One may also have $m_{\sqcap}=m_{\sqcup}$. This is the case, for instance, of the relation in (15), see Appendix $\mathrm{A}(\mathrm{g})$. Note that for this relation, all the reflexive points are above $m_{\sqcup}\left(=m_{\sqcap}=\perp\right)$ and that they are not linearly ordered.

It is even possible to have $m_{\sqcup}<m_{\sqcap}$. Consider for instance the relation $\{(\top, \top)\}$ on the lattice $\{\perp, \top\}$, for which $m_{\sqcap}=\top$ and $m_{\sqcup}=\perp$.

However, for total $R$ we have $\{x \mid \sqcup x R \leq x\} \subseteq\{x \mid \sqcap x R \leq x\}$ and hence $m_{\sqcap} \leq m_{\sqcup}$.

\subsection{Lattice Structure of the Reflexive Points}

We now come to the main result of this section, viz. the generalization of Tarski's result [10] on the fixed points of a monotonic total function to the relational case.

Theorem 3 Let $R$ be a total relation and assume Condition 4(c). Then the set of reflexive points of $R$ is a complete lattice.

Moreover, we have

Proposition 6 For a total relation $R$ satisfying Condition $4(\mathrm{c})$ define $l:=\prod\{x \mid x R x\}$. Then $m_{\sqcap} \leq l \leq m_{\sqcup}$.

Finally, combining our results for the partial case with Theorem 3 , we obtain

Proposition 7 Let $R$ be a partial relation that satisfies $4(\mathrm{a}, \mathrm{b}, \mathrm{c})$ and $\top R \neq \emptyset$. Then the set of reflexive points is a complete lattice.

The condition $T R \neq \emptyset$ is mandatory: the dual of Appendix A(a) satisfies 4(a,b,c) but has no greatest reflexive point, so that the set of reflexive points is not a complete lattice.

Unfortunately, the combination $4(\mathrm{a}, \mathrm{b}, \mathrm{d})$ does not guarantee a complete lattice of reflexive points, not even when $T R \neq \emptyset$. This is shown in the example of Appendix $\mathrm{A}(\mathrm{n})$ : the lattice is $\{\perp, \mathrm{a}, \mathrm{b}, \mathrm{c}, \mathrm{d}, \mathrm{e}, \mathrm{f}, \mathrm{g}, \mathrm{h}, \mathrm{i}, \mathrm{T}\}$, with ordering $\perp<\mathrm{a}<\mathrm{T}, \perp<\mathrm{b}<\mathrm{c}<\mathrm{e}<\mathrm{f}<\mathrm{g}<\mathrm{i}<\mathrm{T}$ and $\mathrm{b}<\mathrm{d}<\mathrm{e}<\mathrm{f}<\mathrm{h}<\mathrm{i}$, and the relation is

$$
\begin{array}{r}
\{(\perp, \perp),(b, c),(b, d),(c, c),(c, d),(d, c),(d, d),(e, c),(e, d), \\
(f, g),(f, h),(g, g),(g, h),(h, g),(h, h),(i, g),(i, h),(\top, \top)\} .
\end{array}
$$

The set $\{\perp, \mathrm{c}, \mathrm{d}, \mathrm{g}, \mathrm{h}, \top\}$ of reflexive points is not a lattice, since, e.g., the subset $\{\mathrm{c}, \mathrm{d}\}$ has two minimal upper bounds, namely g and h. Note how the total relation of Appendix A(l), which also satisfies $4(\mathrm{a}, \mathrm{b}, \mathrm{d})$, is used twice as a sublattice of the partial relation of Appendix $\mathrm{A}(\mathrm{n})$.

\section{Greatest Reflexive Points}

We can obtain results for greatest reflexive points using properties of the least reflexive points in the dual of the given lattice. It suffices to replace $\leq, \sqcap, \sqcup$ and "least" by $\geq, \sqcup, \sqcap$ and "greatest", respectively. Doing so reveals that Conditions 4(a), 4(b), 4(c) and 4(d) are dual to $4(\mathrm{~d}), 4(\mathrm{~b}), 4(\mathrm{c})$ and $4(\mathrm{a})$, respectively. Properties of total or partial relations then easily follow from the previous text.

By Proposition 2, the set of Properties 4(a,b,c) guarantees the existence of a least reflexive point. Its dual, $4(\mathrm{~b}, \mathrm{c}, \mathrm{d})$, guarantees the existence of a greatest reflexive point. The question arises whether it is possible to have a relation satisfying $4(\mathrm{~b}, \mathrm{c}, \mathrm{d})$, and thus having a greatest reflexive point, that does not have a least reflexive point. The answer is yes and is illustrated by the relation given in (6) and Appendix $\mathrm{A}(\mathrm{a})$. 


\section{Can Monotonicity be Characterized Another Way?}

Since we are working in a relational setting, an obvious question is whether monotonicity can be characterized in a purely algebraic, point-free style.

\subsection{Candidate Conditions and Their Interrelation}

For the case of total functions it is well known how to do this; when $R$ is a total function, the following four conditions $20\left(\mathrm{a}^{\prime}, \mathrm{b}^{\prime}, \mathrm{c}^{\prime}, \mathrm{d}^{\prime}\right)$ all are equivalent to the usual pointwise definition of monotonicity. But for the same reasons as in discussed in Section 3.2, we will work with Conditions 20(a,b,c,d) (see also Section 6.2):

$$
\begin{aligned}
& \text { (a) } \quad<; R \subseteq R ; \leq, \quad \text { (a') } \quad \leq ; R \subseteq R ; \leq \text {, } \\
& \text { (b) } \quad<\subseteq R ; \leq ; R^{\cup}, \quad\left(\mathrm{b}^{\prime}\right) \quad \leq \subseteq R ; \leq ; R^{\cup} \text {, } \\
& \text { (c) } R^{\cup} ;<; R \subseteq \leq, \quad \text { (c') } R^{\cup} ; \leq ; R \subseteq \leq \text {, } \\
& \text { (d) } \quad R^{\cup} ;<\subseteq \leq ; R^{\smile}, \quad\left(\mathrm{d}^{\prime}\right) \quad R^{\cup} ; \leq \subseteq \leq ; R^{\smile} \text {. }
\end{aligned}
$$

However, in the case of general relations they are not equivalent. There, we have that Conditions 20(a',b',c',d') are equivalent to the following pointwise expressions.

$$
\begin{array}{ll}
20 \text { (a') } & \forall(x, y: x \leq y: \forall(v: y R v: \exists(u: x R u: u \leq v))) \\
20 \text { (b') } & \forall(x, y: x \leq y: \exists(u, v: x R u \text { and } y R v: u \leq v)) \\
20 \text { (c') } & \forall(x, y: x \leq y: \forall(u, v: x R u \text { and } y R v: u \leq v)) \\
20 \text { (d') } & \forall(x, y: x \leq y: \forall(u: x R u: \exists(v: y R v: u \leq v)))
\end{array}
$$

On the basis of the structure of these expressions, one could say that Conditions 20(a',b',c',d') respectively define upward existential monotonicity, existential monotonicity, universal monotonicity and downward existential monotonicity.

A first consequence of these properties is stated in

Proposition $820(\mathrm{a}, \mathrm{d}) \Rightarrow R=\emptyset$ or $R$ total.

\subsection{Interdependence of the Conditions}

As in the case of our earlier Conditions 4, we state the connections between the unprimed and primed versions

$$
\begin{aligned}
& 20\left(\mathrm{a}^{\prime}\right) \Leftrightarrow 20(\mathrm{a}), \quad 20\left(\mathrm{c}^{\prime}\right) \Leftrightarrow 20(\mathrm{c}) \text { and } R \text { functional, } \\
& 20\left(\mathrm{~b}^{\prime}\right) \Leftrightarrow 20(\mathrm{~b}) \text { and } R \text { total, } 20\left(\mathrm{~d}^{\prime}\right) \Leftrightarrow 20(\mathrm{~d}) \text {. }
\end{aligned}
$$

Hence, the situation is almost identical to that with Conditions 4 (see (12)), except for the equivalence of 20(b') and 20(b). Rather than using all eight Conditions 20, we will simply use Conditions 20(a,b,c,d) together with totality or functionality, like we did for Conditions 4. One can show (see $[5,6]$ ) that the same implications hold between Conditions 20(a,b,c,d) as between 4(a,b,c,d) (see Equation (5)):

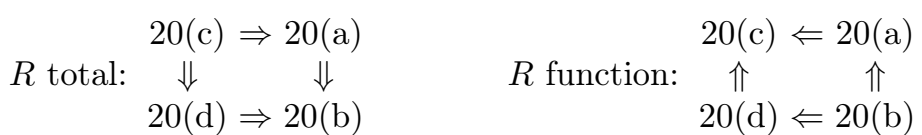

Here too, one can deduce from these diagrams that if $R$ is a total function, all four Conditions 20 (a,b,c,d) are equivalent, as was mentioned in the introductory part of Section 6.1.

In view of these striking similarities, one might expect that corresponding properties in $4(\mathrm{a}, \mathrm{b}, \mathrm{c}, \mathrm{d})$ and $20(\mathrm{a}, \mathrm{b}, \mathrm{c}, \mathrm{d})$ are equivalent. However, this is not the case, as the following theorem shows.

Theorem 4 The following relationships hold between Conditions 4 and 20. All implications are strict (i.e., equivalence does not hold):

$$
\begin{aligned}
& 20(a) \Rightarrow 4(a) \quad 20(b) \Rightarrow 4(b) \\
& 20(c) \Leftrightarrow 4(c) \quad 20(d) \Rightarrow 4(d)
\end{aligned}
$$

A single example (see Appendix $\mathrm{A}(\mathrm{k})$ ) can be used to show that the three implications are strict: Take $S$ to be the lattice $\{\perp, \mathrm{a}, \mathrm{b}, \mathrm{c}, \mathrm{d}, \top\}$ with ordering $\perp<\mathrm{a}<\mathrm{c}<\top$ and $\perp<\mathrm{b}<\mathrm{d}<\mathrm{T}$, and

$$
R:=\{(\perp, \mathrm{c}),(\perp, \mathrm{d}),(\mathrm{a}, \mathrm{c}),(\mathrm{a}, \mathrm{d}),(\mathrm{b}, \mathrm{c}),(\mathrm{b}, \mathrm{d}),(\mathrm{c}, \mathrm{a}),(\mathrm{c}, \mathrm{b}),(\mathrm{d}, \mathrm{a}),(\mathrm{c}, \mathrm{b}),(\mathrm{T}, \mathrm{a}),(\mathrm{T}, \mathrm{b})\} .
$$

This total relation satisfies all of $4(\mathrm{a}, \mathrm{b}, \mathrm{d})$ and none of $20(\mathrm{a}, \mathrm{b}, \mathrm{d})$. The partial relation given in (17) is another example showing the strictness of the implications.

Hence, the question whether these conditions are independent cannot be reduced to our independence results in Section 3. A separate investigation shows the following: 
1. Condition 20(b) does not follow from 20(a,c,d). This is shown by the relation given in (7) (Appendix A(b)).

2. Condition 20 (c) does not follow from $20(\mathrm{a}, \mathrm{b}, \mathrm{d})$. This is shown by the lattice $\{\perp, \mathrm{a}, \top\}$ with ordering $\perp<\mathrm{a}<\top$ and the relation (see Appendix A(m))

$$
R:=\{(\perp, \mathrm{a}),(\perp, \top),(\mathrm{a}, \mathrm{a}),(\mathrm{a}, \top),(\top, \top)\} .
$$

3. Conditions 20(a,d) both follow from $20(\mathrm{~b}, \mathrm{c})$.

4. Conditions 20(a,d) imply 20(b) or 20(c).

Thus the independence properties of Conditions 20 are different from those of Conditions 4 and one cannot have combinations like $20(a, b, c)$ and $20(b, c, d)$ without having all of $20(a, b, c, d)$. However, all the remaining combinations of our properties can be exemplified with lattices and relations, see Appendix B.

\subsection{On the Existence of Least Reflexive Elements}

Do the algebraic Conditions 20 ensure the existence of least reflexive elements of partial relations in the same manner as Conditions 4? Unfortunately, this is not the case, as can be seen as follows. The example in (7) (Appendix A(b)) satisfies 20(a,c,d), but has no reflexive point, so that 20 (b) is essential for the existence.

On the other hand, it can be shown that 20(b) implies totality of $R$. In sum, all this means that Conditions 20 are not useful for studying the reflexive points of partial relations.

For total relations, we have concluded the corresponding Section 3.3 with the remark that Condition 4(c) in Theorem 2 cannot be relaxed to a weaker combination of some of 4(a,b,d). Because Conditions 20(a,b,d) are stronger than 4(a,b,d) (see Theorem 4), one might conjecture that 4(c) (equivalently, 20(c)) could be weakened to a combination of some of $20(\mathrm{a}, \mathrm{b}, \mathrm{d})$. However, this is not the case. The lattice $\{\perp, \mathrm{a}, \mathrm{b}, \top\}$, with ordering $\perp<\mathrm{a}<\top$ and $\perp<\mathrm{b}<\mathrm{T}$, and the relation

$$
R:=\{(\perp, \mathrm{a}),(\perp, \mathrm{b}),(\mathrm{a}, \mathrm{a}),(\mathrm{a}, \mathrm{b}),(\mathrm{b}, \mathrm{a}),(\mathrm{b}, \mathrm{b}),(\top, \mathrm{a}),(\top, \mathrm{b})\}
$$

(see Appendix A(l)) provide an example of a total relation that satisfies all Conditions $20(\mathrm{a}, \mathrm{b}, \mathrm{d})$ and that has no least reflexive point.

\subsection{Other Conditions Ensuring Reflexive Elements}

In this paper we have solely treated the case of complete lattices. However, it is well known that under suitable assumptions Tarski's original fixed point theorem carries over to weaker structures such as chain-complete partial orders (cpo's).

In this connection we want to mention the paper [7] by Fujimoto. It studies total relations, represented as set-valued functions, on a cpo with a least element. The monotonicity condition used is $20\left(\mathrm{~d}^{\prime}\right)$. Fujimoto shows that under the additional assumption that all image sets under $R$ are inductively ordered, i.e., contain for each subchain an upper bound, there is an $R$-reflexive element, although not necessarily a least one.

It will be interesting to investigate further sufficient conditions with weaker assumptions.

\section{Conclusion}

This paper provides a first survey on the structure of the set of reflexive points of relations on complete lattices. We have exhibited suitable adaptations of the notion of monotonicity of a total function to the relational case. It may come as a certain surprise that, unless other conditions are added (as in Section 6.4), the direct relational formulations of monotonicity of a total function are of no use in this setting and have to be replaced by new conditions. With the help of these we have shown an analogue of Tarski's classical result [10] on existence and lattice structure of the reflexive points. Another advantage of the new conditions is that they are checked manually much more easily than the relational ones. What is still missing is a suitable generalization of the notion of continuity and, following that, an investigation when least reflexive elements can be obtained by iteration as in Kleene's Theorem [8] (the process being also already mentioned in [10]).

Another open question in connection with iteration (see Section 2) is whether there are (not too strong) conditions guaranteeing the existence of a least reflexive point when the strict ordering is progressively finite and the relation is inflationary. Moreover, which 
additional conditions ensure that any path by $R$ from $\perp$ leads to this least reflexive point? We conclude this paper with a last counter-example showing that Conditions $4(\mathrm{a}, \mathrm{b}, \mathrm{d})$ are not sufficient to guarantee this. Consider again the relation $R$ from (24) (see Appendix A(m)). That relation is inflationary and satisfies $4(\mathrm{a}, \mathrm{b}, \mathrm{d})$, the strict ordering is progressively finite, there is a least and a greatest reflexive point, but there is a path from $\perp$ to $\top$ that does not go through the least reflexive point, which is a.

\section{Acknowledgements}

We are grateful to the referees of paper [6] for their helpful remarks, in particular to Oege de Moor for his hint to look at Condition 20(a). Valuable comments were also provided by Thorsten Ehm and Georg Struth.

\section{A Examples and Counterexamples}

This section contains diagrams of some of the lattices and relations presented in the paper. Please see overleaf for further explanation.

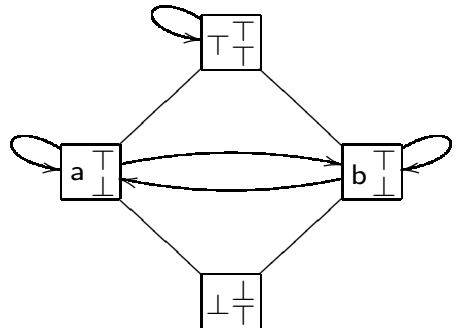

a) $4(\mathrm{~b}, \mathrm{c}, \mathrm{d})$ $20(\mathrm{c}, \mathrm{d})$ Equation 6

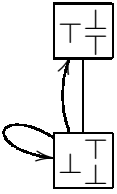

d) $4(\mathrm{a}, \mathrm{b}, \mathrm{c})$ $20(\mathrm{a}, \mathrm{c})$ Equation 9

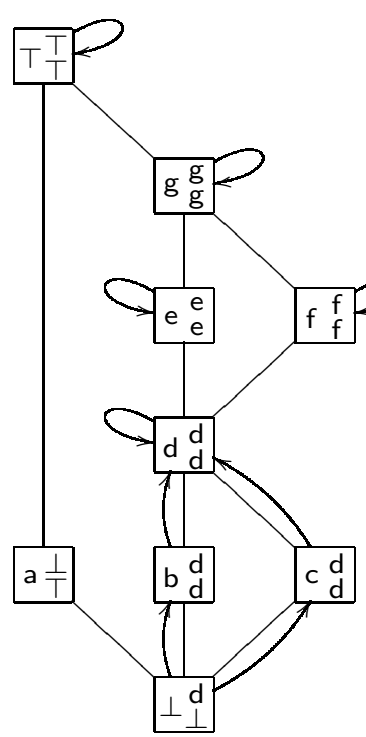

g) $4(\mathrm{a}, \mathrm{b}, \mathrm{c})$

$20(\mathrm{c})$

Equation 15

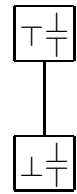

b) $4(\mathrm{a}, \mathrm{c}, \mathrm{d})$ $20(a, c, d)$ Equation 7

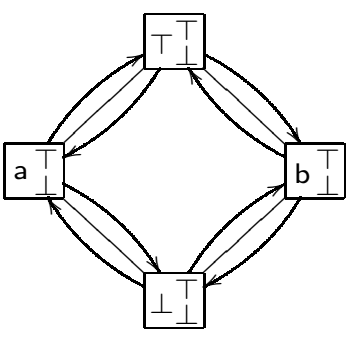

c) $4(\mathrm{a}, \mathrm{b}, \mathrm{d})$ $20(\mathrm{~b})$

Equation 8
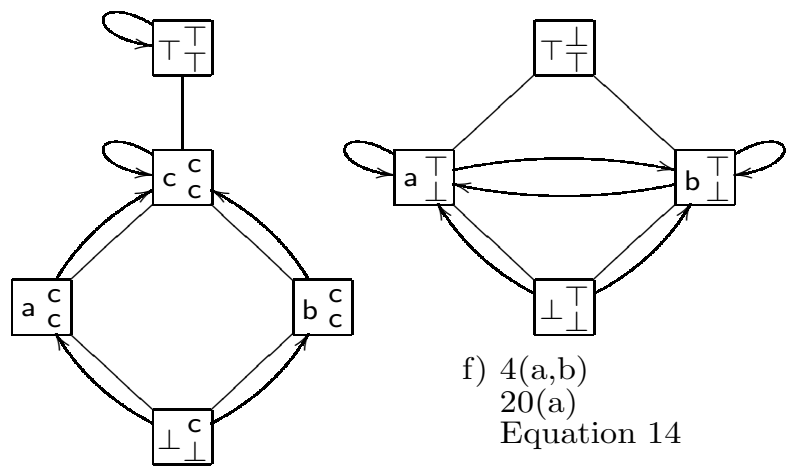

f) $4(\mathrm{a}, \mathrm{b})$

20(a)

Equation 14

e) $4(\mathrm{a}, \mathrm{b}, \mathrm{c}, \mathrm{d})$

$20(a, b, c, d)$

Inflationary

Equation 13

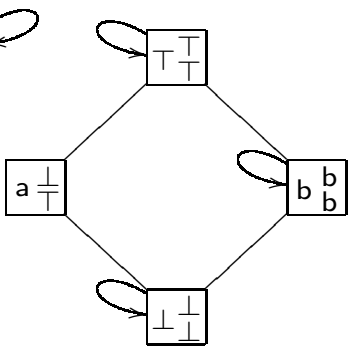

h) $4(\mathrm{a}, \mathrm{b}, \mathrm{c}, \mathrm{d})$ 20 (c)

Equation 16

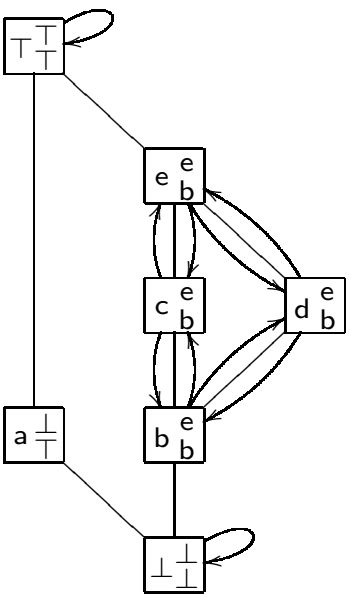

i) $4(\mathrm{a}, \mathrm{b}, \mathrm{d})$

Equation 17 
Each lattice $(S, \sqcap, \sqcup, \perp, \top, \leq)$ is described by the Hasse diagram of its corresponding partial order, with boxes representing vertices (elements of $S$ ) and straight lines representing edges. The relation $R$ on the lattice is represented by arrows linking the boxes. Each box contains three pieces of information: (i) on the left is the element $s \in S$, (ii) on the bottom right is $\Pi s R$, and (iii) on the top right is $\sqcup s R$. The following information is given under each diagram: (i) the list of properties among $4(\mathrm{a}, \mathrm{b}, \mathrm{c}, \mathrm{d})$ that hold for this diagram, (ii) the list of properties among 20(a,b,c,d) that hold for this diagram, (iii) the word "Inflationary", if the relation is inflationary, and (iv) the equation where the relation is defined.

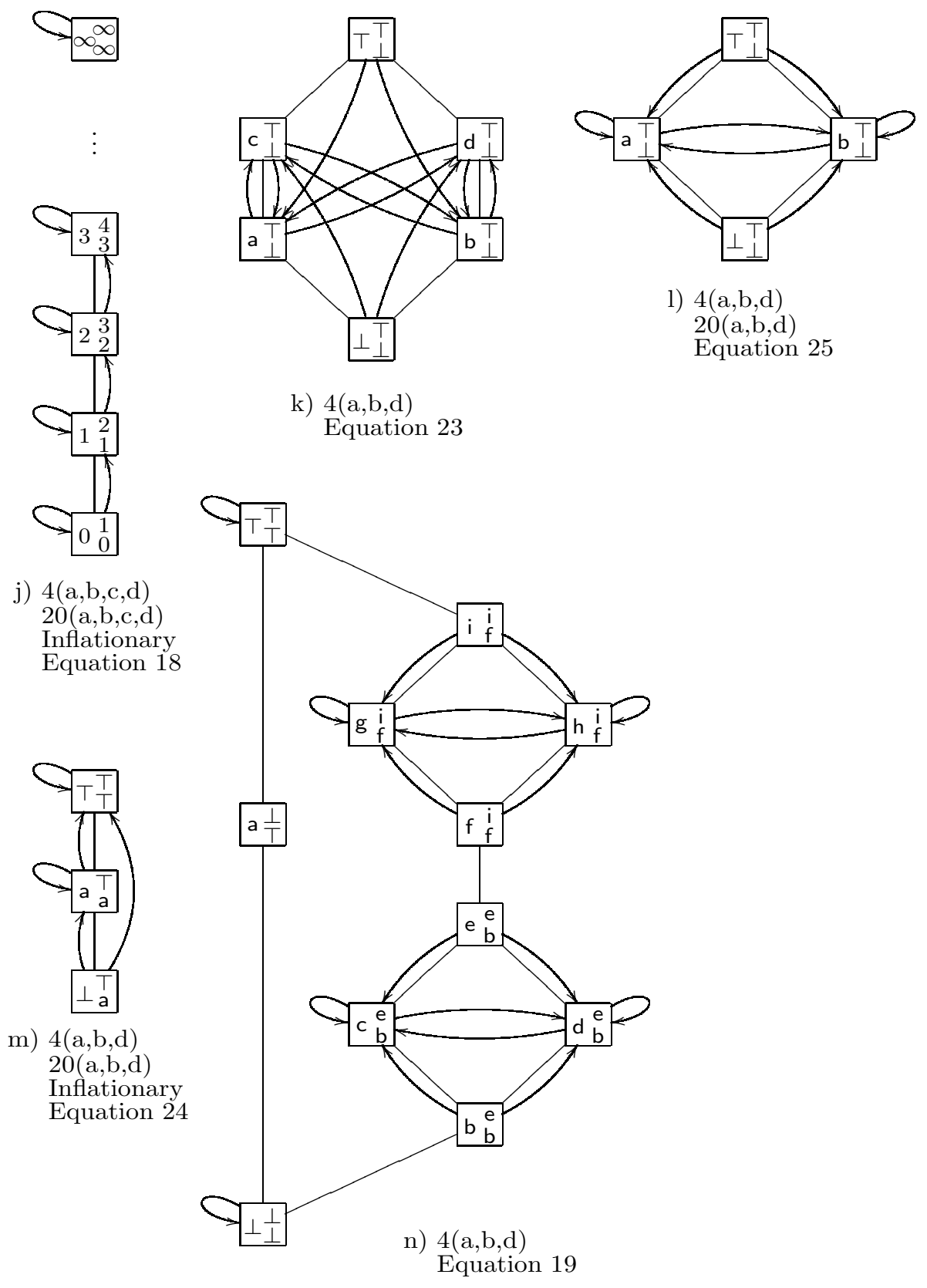

\section{B Combinations of Properties}

Here are examples of lattices and relations showing all possible combinations of Conditions 4 and all possible combinations of Conditions 20. For all but one example, there is no need of sophisticated lattices: two linear orders suffice! Each example uses a minimal lattice, except 
possibly relation (p) in the tables below; also, the trivial lattice with $T=\perp$ with the empty or the universal relation could be used instead of $(\mathrm{j})$ to illustrate $4(\mathrm{a}, \mathrm{b}, \mathrm{c}, \mathrm{d})$ and $20(\mathrm{a}, \mathrm{b}, \mathrm{c}, \mathrm{d})$.

The labels of the following relations correspond to those of the diagrams that follow the presentation of the relations.

1. Lattice $\{\perp, \top\}$ with ordering $\perp<\top$.

\begin{tabular}{l|l|l|}
\multicolumn{2}{c}{ Relation } & \multicolumn{1}{l}{ Properties } \\
\cline { 2 - 3 } (a) & $\{(\perp, \top),(\top, \perp)\}$ & None \\
(b) & $\{(\perp, \perp),(\perp, \top),(\top, \perp)\}$ & $4(\mathrm{a}, \mathrm{b}), 20(\mathrm{a}, \mathrm{b})$ \\
(c) & $\{(\perp, \top)\}$ & $4(\mathrm{a}, \mathrm{c}), 20(\mathrm{a}, \mathrm{c})$ \\
(d) & $\{(\perp, \top),(\top, \perp),(\top, \top)\}$ & $4(\mathrm{~b}, \mathrm{~d}), 20(\mathrm{~b}, \mathrm{~d})$ \\
(e) & $\{(\top, \perp)\}$ & $4(\mathrm{c}, \mathrm{d}), 20(\mathrm{c}, \mathrm{d})$ \\
(f) & $\{(\perp, \perp),(\perp, \top)\}$ & $4(\mathrm{a}, \mathrm{b}, \mathrm{c}), 20(\mathrm{a}, \mathrm{c})$ \\
(g) & $\{(\perp, \perp),(\perp, \top),(\top, \perp),(\top, \top)\}$ & $4(\mathrm{a}, \mathrm{b}, \mathrm{d}), 20(\mathrm{a}, \mathrm{b}, \mathrm{d})$ \\
(h) & $\emptyset$ & $4(\mathrm{a}, \mathrm{c}, \mathrm{d}), 20(\mathrm{a}, \mathrm{c}, \mathrm{d})$ \\
(i) & $\{(\top, \perp),(\top, \top)\}$ & $4(\mathrm{~b}, \mathrm{c}, \mathrm{d}), 20(\mathrm{c}, \mathrm{d})$ \\
(j) & $\{(\perp, \perp),(\top, \top)\}$ & $4(\mathrm{a}, \mathrm{b}, \mathrm{c}, \mathrm{d}), 20(\mathrm{a}, \mathrm{b}, \mathrm{c}, \mathrm{d})$ \\
&
\end{tabular}

2. Lattice $\{\perp, \mathrm{a}, \top\}$ with ordering $\perp<\mathrm{a}<\top$.

\begin{tabular}{l|l|l|}
\multicolumn{1}{c}{ Relation } & \multicolumn{1}{c}{ Properties } \\
\cline { 2 - 3 }$(\mathrm{k})$ & $\{(\perp, \perp),(\perp, \top),(\mathrm{a}, \mathrm{a})\}$ & $4(\mathrm{a}), 20(\mathrm{a})$ \\
$(\mathrm{l})$ & $\{(\perp, \mathrm{a}),(\perp, \top),(\mathrm{a}, \perp),(\mathrm{a}, \mathrm{a}),(\top, \mathrm{a})\}$ & $4(\mathrm{~b}), 20(\mathrm{~b})$ \\
$(\mathrm{m})$ & $\{(\mathrm{a}, \perp),(\mathrm{a}, \mathrm{a})\}$ & $4(\mathrm{c}), 20(\mathrm{c})$ \\
(n) & $\{(\mathrm{a}, \mathrm{a}),(\top, \perp),(\top, \top)\}$ & $4(\mathrm{~d}), 20(\mathrm{~d})$ \\
(o) & $\{(\perp, \perp),(\perp, \mathrm{a}),(\top, \mathrm{a}),(\top, \top)\}$ & $4(\mathrm{~b}, \mathrm{c}), 20(\mathrm{c})$ \\
\cline { 2 - 3 } & &
\end{tabular}

3. Lattice $\{\perp, \mathrm{a}, \mathrm{b}, \mathrm{c}, \mathrm{d}, \top\}$ with ordering $\perp<\mathrm{a}<\mathrm{c}<\top$ and $\perp<\mathrm{b}<\mathrm{d}<\top$. Relation Properties

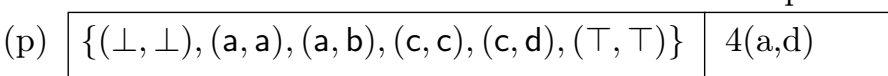

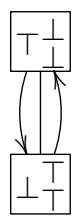

a) None

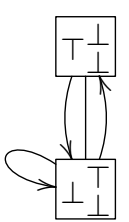

b) $4(\mathrm{a}, \mathrm{b})$ $20(a, b)$

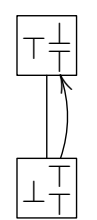

c) $4(\mathrm{a}, \mathrm{c})$ $20(a, c)$

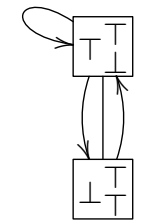

d) $4(\mathrm{~b}, \mathrm{~d})$ $20(\mathrm{~b}, \mathrm{~d})$

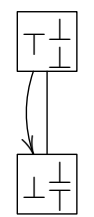

e) $4(\mathrm{c}, \mathrm{d})$ $20(\mathrm{c}, \mathrm{d})$

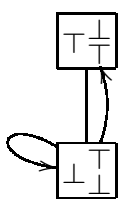

f) $4(\mathrm{a}, \mathrm{b}, \mathrm{c})$ $20(\mathrm{a}, \mathrm{c})$

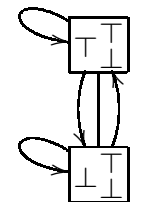

g) $4(\mathrm{a}, \mathrm{b}, \mathrm{d})$ $20(a, b, d)$

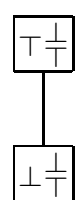

h) $4(\mathrm{a}, \mathrm{c}, \mathrm{d})$ $20(a, c, d)$

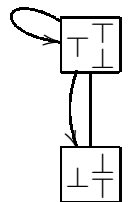

i) $4(\mathrm{~b}, \mathrm{c}, \mathrm{d})$ $20(\mathrm{c}, \mathrm{d})$

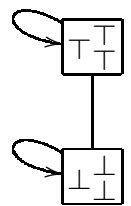

j) $4(\mathrm{a}, \mathrm{b}, \mathrm{c}, \mathrm{d})$ $20(a, b, c, d)$ 


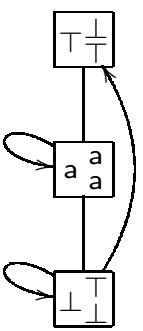

k) $4(\mathrm{a})$ $20(a)$

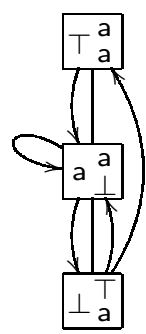

1) $4(\mathrm{~b})$ $20(\mathrm{~b})$

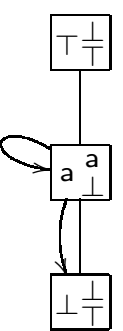

m) $4(\mathrm{c})$

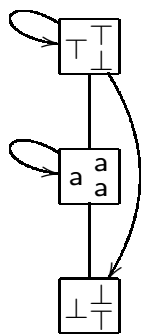

n) $4(\mathrm{~d})$ $20(d)$

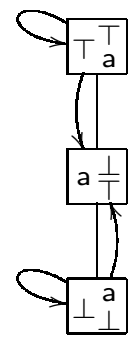

o) $4(\mathrm{~b}, \mathrm{c})$ $20(c)$

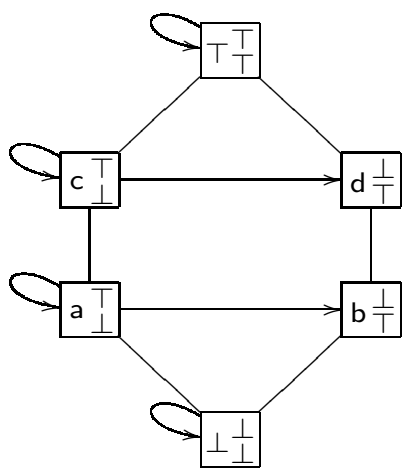

p) $4(\mathrm{a}, \mathrm{d})$

\section{References}

1. R. Backhouse et al.: Fixed point calculus. Information Processing Letters 53(3), 131-136, 1995.

2. J. Cai and R. Paige: Program derivation by fixed point computation. Science of Computer Programming 11(3), 197-261, 1989.

3. J. Cai and R. Paige: Languages polynomial in the input plus output. In: Second International Conference on Algebraic Methodology and Software Technology (AMAST '91), London, Springer, 287-300, 1992.

4. B. A. Davey and H. A. Priestley: Introduction to Lattices and Order. Cambridge Mathematical Textbooks. Cambridge University Press, Cambridge, 1990.

5. J. Desharnais and B. Möller: Least reflexive points of relations. Research Report, Institut für Informatik, Universität Augsburg, Germany, 2002.

6. J. Desharnais and B. Möller: Least reflexive points of relations. Higher-Order and Symbolic Computation. Special Issue in memory of Robert Paige. 18(1/2), 51-77, 2005.

7. T. Fujimoto: An extension of Tarski's fixed point theorem and its application to isotone complementarity problems. Mathematical Programming 28, 116-118, 1984.

8. S. C. Kleene: Introduction to Metamathematics. Van Nostrand, New York, 1952.

9. G. Schmidt and T. Ströhlein: Relations and Graphs. EATCS Monographs in Computer Science. Springer-Verlag, Berlin, 1993.

10. A. Tarski: A lattice-theoretical fixpoint theorem and its applications. Pacific Journal of Mathematics 5, 285-309, 1955. 